\title{
Comprehensive mapping of immune tolerance yields a regulatory TNF receptor 2 signature in a murine model of successful Fel d 1-specific immunotherapy using high-dose $\mathrm{CpG}$ adjuvant
}

\author{
Cathy Leonard ${ }^{1}$, Guillem Montamat ${ }^{1}$, Caroline Davril ${ }^{1}$, Olivia Domingues ${ }^{1}$, Oliver \\ Hunewald $^{1}$, Dominique Revets ${ }^{1}$, Coralie Guerin ${ }^{1}$, Simon Blank ${ }^{2}$, Justine Heckendorn ${ }^{1}$, \\ Gauthier Jardon ${ }^{1}$, François Hentges ${ }^{1}$, and Markus Ollert ${ }^{3}$ \\ ${ }^{1}$ Luxembourg Institute of Health Department of Infection and Immunity \\ ${ }^{2}$ School of Medicine and Helmholtz Center Munich \\ ${ }^{3}$ Luxembourg Institute of Health
}

July 24, 2020

\begin{abstract}
Background The prevalence of allergy to cat is expanding worldwide. Allergen-specific immunotherapy (AIT) has advantages over symptomatic pharmacotherapy and promises long lasting disease control in allergic patients. However, there is still a need to improve cat AIT regarding efficacy, safety and adherence to the treatment. Here we aim to boost immune tolerance to the major cat allergen Fel d 1 by increasing the anti-inflammatory activity of AIT with the established immunomodulatory adjuvant $\mathrm{CpG}$, but at a higher dose than previously used in AIT. Methods Together with CpG, we used endotoxin-free Fel d 1 as therapeutic allergen throughout the study in a BALB/c model of allergy to Fel d 1, thus mimicking the conditions of human AIT trials. Multidimensional immune phenotyping including mass cytometry was applied to analyze AIT-specific immune signatures. Results We show that AIT with high-dose CpG in combination with endotoxin-free Fel d 1 reverts all major hallmarks of allergy. High dimensional CyTOF analysis of the immune cell signatures initiating and sustaining the AIT effect indicates the simultaneous engagement of both, the pDC-Treg and -B cell axis, with the emergence of a systemic GATA3+ FoxP3hi biTreg population. The regulatory immune signature also suggests the involvement of the anti-inflammatory TNF/TNFR2 signaling cascade in NK and B cells at an early stage and in Tregs later during AIT. Conclusion Our results highlight the potential of $\mathrm{CpG}$ adjuvant in a novel formulation to be further exploited for inducing allergen-specific tolerance in patients with cat allergy or other allergic diseases in the future.
\end{abstract}

\section{Hosted file}

Main text and figures Leonard c et al..pdf available at https://authorea.com/users/345786/ articles/471906-comprehensive-mapping-of-immune-tolerance-yields-a-regulatory-tnfreceptor-2-signature-in-a-murine-model-of-successful-fel-d-1-specific-immunotherapyusing-high-dose-cpg-adjuvant 